\title{
Front Matter: Volume 7728
}

, "Front Matter: Volume 7728," Proc. SPIE 7728, Nonlinear Optics and Applications IV, 772801 (16 June 2010); doi: 10.1117/12.867178

SPIE. Event: SPIE Photonics Europe, 2010, Brussels, Belgium 


\title{
PROCEEDINGS OF SPIE
}

\section{Nonlinear Optics and Applications IV}

\author{
Benjamin J. Eggleton \\ Alexander Luis Gaeta \\ Neil G. R. Broderick \\ Editors
}

12-15 April 2010

Brussels, Belgium

Sponsored by

SPIE

Cosponsored By

B-PHOT_Brussels Photonics Team (Belgium) - Brussels-Capital Region (Belgium) • FWO_Fonds Wetenschappelijk Onderzoek (Belgium) • ICO_International Commission for Optics • Ville de Bruxelles (Belgium)

Cooperating Organisations

CBO-BCO (Belgium) • EOS-European Optical Society (Germany) • IET-The Institution of Engineering and Technology (United Kingdom) $\bullet$ IOP-Institute of Physics (United Kingdom) Photonics4Life (Germany) • Photonics@be (Belgium) - Photonics 21 (Germany) • PromOptica (Belgium)

Published by

SPIE

Volume 7728 
The papers included in this volume were part of the technical conference cited on the cover and title page. Papers were selected and subject to review by the editors and conference program committee. Some conference presentations may not be available for publication. The papers published in these proceedings reflect the work and thoughts of the authors and are published herein as submitted. The publisher is not responsible for the validity of the information or for any outcomes resulting from reliance thereon.

Please use the following format to cite material from this book:

Author(s), "Title of Paper," in Nonlinear Optics and Applications IV, edited by Benjamin J. Eggleton, Alexander Luis Gaeta, Neil G. R. Broderick, Proceedings of SPIE Vol. 7728 (SPIE, Bellingham, WA, 2010) Article CID Number.

ISSN 0277-786X

ISBN 9780819482013

Published by

SPIE

P.O. Box 10, Bellingham, Washington 98227-0010 USA

Telephone +1 3606763290 (Pacific Time) · Fax +1 3606471445

SPIE.org

Copyright (C) 2010, Society of Photo-Optical Instrumentation Engineers

Copying of material in this book for internal or personal use, or for the internal or personal use of specific clients, beyond the fair use provisions granted by the U.S. Copyright Law is authorized by SPIE subject to payment of copying fees. The Transactional Reporting Service base fee for this volume is $\$ 18.00$ per article (or portion thereof), which should be paid directly to the Copyright Clearance Center (CCC), 222 Rosewood Drive, Danvers, MA 01923. Payment may also be made electronically through CCC Online at copyright.com. Other copying for republication, resale, advertising or promotion, or any form of systematic or multiple reproduction of any material in this book is prohibited except with permission in writing from the publisher. The CCC fee code is 0277-786X/10/\$18.00.

Printed in the United States of America.

Publication of record for individual papers is online in the SPIE Digital Library.

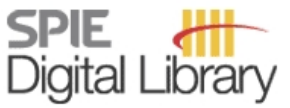

SPIEDigitalLibrary.org

Paper Numbering: Proceedings of SPIE follow an e-First publication model, with papers published first online and then in print and on CD-ROM. Papers are published as they are submitted and meet publication criteria. A unique, consistent, permanent citation identifier (CID) number is assigned to each article at the time of the first publication. Utilization of CIDs allows articles to be fully citable as soon they are published online, and connects the same identifier to all online, print, and electronic versions of the publication. SPIE uses a six-digit CID article numbering system in which:

- The first four digits correspond to the SPIE volume number.

- The last two digits indicate publication order within the volume using a Base 36 numbering system employing both numerals and letters. These two-number sets start with 00, 01, 02, 03, 04, $05,06,07,08,09,0 A, 0 B \ldots 0 Z$, followed by 10-1Z, 20-2Z, etc.

The CID number appears on each page of the manuscript. The complete citation is used on the first page, and an abbreviated version on subsequent pages. Numbers in the index correspond to the last two digits of the six-digit CID number. 


\section{Contents}

ix Conference Committee

SESSION 1 PAST AND FUTURE OF NONLINEAR OPTICS

772803 Optical sampling of ultrahigh bitrate signals using highly nonlinear chalcogenide planar waveguides or tapered fibers [7728-02]

J. Van Erps, The Univ. of Sydney (Australia) and Vrije Univ. Brussel (Belgium); F. Luan, M. D. Pelusi, E. Mägi, T. Iredale, The Univ. of Sydney (Australia); S. Madden, D.-Y. Choi, D. A. Bulla, B. Luther-Davies, The Australian National Univ. (Australia); H. Thienpont, Vrije Univ. Brussel (Belgium); B. J. Eggleton, The Univ. of Sydney (Australia)

772804 Tunable, repetition rate selective, passive mode-locked fibre laser with repetition rates up to $640 \mathrm{GHz}$ [7728-03]

J. Schröder, T. D. Vo, B. J. Eggleton, The Univ. of Sydney (Australia)

772805 High repetition rate pulse train generation at $\mathrm{GHz}$ repetition rates from nonlinear breather reshaping in standard single mode fibre [7728-04]

R. Maher, P. M. Anandarajah, L. P. Barry, Dublin City Univ. (Ireland); B. Wetzel, J. M. Dudley, Institut FEMTO-ST, CNRS, Univ. de Franche-Comté (France)

\section{SESSION 2 SEMICONDUCTOR BASED NONLINEAR}

772807 Silicon based ultrafast optical waveform sampling (Best Student Paper Award) [7728-06] H. Ji, M. Galili, M. Pu, L. K. Oxenløwe, P. Jeppesen, Technical Univ. of Denmark (Denmark)

772808 Propagation losses in GaAs/AIOx nonlinear waveguide and their impact on parametric oscillation threshold [7728-07]

E. Guillotel, C. Langlois, M. Savanier, F. Ghiglieno, S. Ducci, I. Favero, G. Leo, Lab. Matériaux et Phénomènes Quantiques, CNRS, Univ. Paris Diderot-Paris 7 (France)

772809 Investigation of on-chip all-optical quantization and novel encoding method: paving the way for optical analog-to-digital conversion chip [7728-08]

R. Pant, C. Xiong, The Univ. of Sydney (Australia); S. Madden, B. Luther Davies, The Australian National Univ. (Australia); B. J. Eggleton, The Univ. of Sydney (Australia)

7728 OB Reservoir computing: a photonic neural network for information processing [7728-10]

Y. Paquot, Univ. Libre de Bruxelles (Belgium); J. Dambre, B. Schrauwen, Univ. Gent (Belgium);

M. Haelterman, S. Massar, Univ. Libre de Bruxelles (Belgium)

\section{SESSION 3 PHOTONIC CRYSTAL FIBRES}

7728 OD Non-linear spectral broadening across multiple bandgaps of all solid photonic crystal fibers [7728-12]

V. Pureur, J. M. Dudley, Institut FEMTO-ST, CNRS, Univ. de Franche-Comté (France) 
$7728 \mathrm{OE}$ Experimental investigation of a parabolic pulse generation using tapered microstructured optical fibres [7728-13]

N. Vukovic, F. Parmigiani, A. Camerlingo, M. Petrovich, P. Petropoulos, N. G. R. Broderick, Univ. of Southampton (United Kingdom)

7728 OF Impact of third-order dispersion on the evolution of parabolic pulses [7728-14]

S. Boscolo, B. G. Bale, Aston Univ. (United Kingdom)

7728 OG Role of dispersion profile in controlling emission of dispersive waves by solitons inside optical fibers [7728-15]

S. Roy, S. K. Bhadra, Central Glass and Ceramic Research Institute (India); G. P. Agrawal, Univ. of Rochester (United States)

\section{SESSION 4 SLOW LIGHT EFFECTS}

7728 0J Chirped pulse amplification in a fiber optical parametric amplifier [7728-18]

C. Caucheteur, Faculté Polytechnique, Univ. de Mons (Belgium); D. Bigourd, E. Hugonnot, Commissariat à l'Énergie Atomique (France); P. Szriftgiser, A. Kudlinski, Lab. PhLAM, CNRS, Univ. des Sciences et Technologies de Lille (France); M. González-Herráez, Univ. de Alcalá de Henares (Spain); A. Mussot, Lab. PhLAM, CNRS, Univ. des Sciences et Technologies de Lille (France)

7728 OK Multi-resonant microresonators for optical frequency conversion [7728-19]

K. Kusiaku, X. Letartre, J. L. Leclercq, P. Rojo-Romeo, C. Seassal, P. Viktorovitch, Institut des Nanotechnologies de Lyon, CNRS, Univ. de Lyon, Ecole Centrale de Lyon (France)

7728 OL Energy density characterization of complex ultrashort laser pulses [7728-20]

A. Lotti, Univ. degli Studi dell'Insubria (Italy), Ctr. de Physique Théorique, CNRS, École Polytechnique (France), and Virtual Institute of Nonlinear Optics (Italy); A. Couairon, Ctr. de Physique Théorique, CNRS, École Polytechnique (France) and Virtual Institute of Nonlinear Optics (Italy); D. Faccio, P. Di Trapani, Univ. degli Studi dell'Insubria (Italy) and Virtual Institute of Nonlinear Optics (Italy)

\section{SESSION $5 \quad$ NEW FRONTIERS IN NONLINEAR OPTICS}

7728 OM Analogue gravity and ultrashort laser pulse filamentation (Invited Paper) [7728-21]

D. Faccio, Univ. degli Studi dell'Insubria (Italy) and Consorzio Nazionale Interuniversitario per le Scienze Fisiche della Materia (Italy); F. Belgiorno, Univ. degli Studi di Milano (Italy); S. Cacciatori, Univ. degli Studi dell'Insubria (Italy) and Istituto Nazionale di Fisica Nucleare (Italy); M. Clerici, Univ. degli Studi dell'Insubria (Italy) and Consorzio Nazionale Interuniversitario per le Scienze Fisiche della Materia (Italy); V. Gorini, Univ. degli Studi dell'Insubria (Italy) and Istituto Nazionale di Fisica Nucleare (Italy); G. Ortenzi, Univ. degli Studi di Milano-Bicocca (Italy); L. Rizzi, E. Rubino, V. G. Sala, Univ. degli Studi dell'Insubria (Italy) 
7728 OR Picoseconds all-optical switch and pulse re-shaper based in a bistable Bragg grating cavity [7728-26]

I. V. Kabakova, C. M. de Sterke, B. J. Eggleton, The Univ. of Sydney (Australia)

7728 OS Time resolved spectral analysis for pulsed lasers nonlinear effects characterization [7728-27]

P. Beaure d'Augères, Lab. Foton, CNRS (France) and Quantel Group (France); A. Mugnier,

D. Pureur, Quantel Group (France); T. Chartier, Lab. Foton, CNRS (France)

7728 OT Time-lens based optical packet pulse compression and retiming [7728-28]

J. L. Areal, H. Hu, E. Palushani, A. T. Clausen, M. S. Berger, L. K. Oxenløwe, Technical Univ. of Denmark (Denmark)

7728 OU Variable optical buffer for packet storage in OPS nodes [7728-29]

G. Berrettini, G. Meloni, Scuola Superiore Sant'Anna (Italy); L. Potì, A. Bogoni, Consorzio

Nazionale Interuniv. per le Telecomunicazioni (Italy)

\section{SESSION 7 PULSE GENERATION AND MANIPULATION}

7728 OW Advection effect in a photorefractive single feedback system: from noise- to dynamicssustained instabilities [7728-31]

N. Marsal, Supélec (France) and Univ. Paul Verlaine de Metz (France); D. Wolfersberger, M. Sciamanna, Supélec (France); G. Montemezzani, Univ. Paul Verlaine de Metz (France)

7728 OY Sum-frequency generation in disordered quadratic nonlinear media [7728-33]

F. Sibbers, J. Imbrock, C. Denz, Westfälische Wilhelms-Univ. Münster (Germany)

$7728 \mathrm{OZ}$ The on-off contrast in an all optical switch based on stimulated Raman scattering in optical fibers [7728-34]

A. Flores-Rosas, E. A. Kuzin, B. Ibarra-Escamilla, Instituto Nacional de Astrofísica, Óptica y Electrónica (Mexico); O. Pottiez, Ctr. de Investigaciones en Óptica, A.C. (Mexico); M. Duran-Sánchez, Univ. Tecnologica de Puebla (Mexico)

772810 Optical rogue waves and stimulated supercontinuum generation (Invited Paper) [7728-35] D. R. Solli, Univ. of California, Los Angeles (United States); C. Ropers, Univ. of California, Los Angeles (United States) and Georg-August-Univ. Göttingen (Germany); B. Jalali, Univ. of California, Los Angeles (United States)

\section{SESSION 8 SUPERCONTINUUM GENERATION}

772811 XUV frequency combs (Invited Paper) [7728-36]

A. Ozawa, A. Vernaleken, I. Gotlibovych, P. Hommelhoff, T. Udem, T. W. Hänsch, Max-Planck-Institut für Quantenoptik (Germany)

$772812 \quad$ Akhmediev Breather dynamics and the nonlinear modulation instability spectrum [7728-37] G. Genty, Tampere Univ. of Technology (Finland); F. Dias, Ecole Normale Supérieure de Cachan (France); B. Kibler, Institut Carnot de Bourgogne, CNRS, Univ. de Bourgogne (France); N. Akhmediev, The Australian National Univ. (Australia); J. M. Dudley, Univ. de Franche-Comté (France) 
772817 Analysis of linear and nonlinear optical properties of diffraction gratings inscribed on the surface of single crystals of the $\mathrm{KTiOPO}_{4}$ family [7728-42]

G. R. Kumar, J. J. Carvajal, M. C. Pujol, X. Mateos, M. Aguiló, F. Diaz, Univ. Rovira i Virgili (Spain); J. R. Vázquez de Aldana, C. Romero, C. Méndez, P. Moreno, L. Roso, Univ. de Salamanca (Spain); J. Ferré-Borrull, J. Pallarès, L. F. Marsal, Univ. Rovira i Virgili (Spain); R. Macovez, J. Martorell, ICFO - Instituto de Ciencias Fotónicas (Spain)

772819 Nonlinear photonic crystals of strontium tetraborate: properties and conversion of radiation [7728-44]

A. S. Aleksandrovsky, Kirensky Institute of Physics (Russian Federation) and Siberian Federal Univ. (Russian Federation); A. M. Vyunishev, Kirensky Institute of Physics (Russian Federation); A. I. Zaitsev, Kirensky Institute of Physics (Russian Federation) and Siberian Federal Univ. (Russian Federation); V. E. Rovsky, V. V. Slabko, Siberian Federal Univ. (Russian Federation); A. V. Cherepakhin, Kirensky Institute of Physics (Russian Federation)

\section{SESSION 10 NOVEL NONLINEAR EFFECTS}

7728 1B Models for coherent anti-Stokes Raman scattering in Raman devices and in spectroscopy [7728-46]

N. Vermeulen, C. Debaes, H. Thienpont, Vrije Univ. Brussel (Belgium)

7728 1C Collision of optical pulses in nonlinear dispersive media: frequency funing and velocity variation [7728-47]

A. P. Sukhorukov, Lomonosov Moscow State Univ. (Russian Federation); V. E. Lobanov, Lomonosov Moscow State Univ. (Russian Federation) and ICFO-Institut de Ciencies Fotoniques, Univ. Politecnica de Catalunya (Spain)

7728 1D Nonlinear interaction of optical beams in gradient waveguides [7728-48]

A. K. Sukhorukova, A. P. Sukhorukov, Lomonosov Moscow State Univ. (Russian Federation)

POSTER SESSION

7728 IE All-optical set-reset flip-flop by nonlinear coupling of microring resonators [7728-50] A. Ghadi, S. Mirzanezhad, F. Sohbatzadeh, Univ. of Mazandaran (Iran, Islamic Republic of)

7728 IF Two photon absorption effect on all-optical semiconductor based switching nonlinear directional coupler [7728-51]

A. Ghadi, S. Mirzanezhad, F. Sohbatzadeh, Univ. of Mazandaran (Iran, Islamic Republic of)

7728 1G Structure optimization of electro-optic polymer waveguides for low half-wave voltage modulators [7728-52]

H. Mahé, D. Bosc, N. Gayet, Lab. Foton, CNRS, Univ. Européenne de Bretagne (France); F. Odobel, Lab. CEISAM, CNRS, Univ. de Nantes (France); P.-A. Bonnardel, Produits Chimiques Auxiliaires et de Synthèse (France); D. M. Nguyen, Lab. Foton, CNRS, Univ. Européenne de Bretagne (France); M. Halbwax, IEMN, CNRS (France) 
$772811 \quad$ Four-input NOR gate using cross gain modulation in a single Semiconductor Optical Amplifier [7728-54]

M. Cabezón, A. Villafranca, J. J. Martínez, D. Izquierdo, I. Garcés, Univ. de Zaragoza (Spain); J. Pozo, Technische Univ. Eindhoven (Netherlands)

7728 1K Terahertz pulse detection by the GaAs Schottky diodes [7728-56]

T. Laperashvili, O. Kvitsiani, I. Imerlishvili, Institute of Cybernetics (Georgia); D. Laperashvili, Georgian Technical Univ. (Georgia)

7728 iN Phthalocyanines for photonic applications: a new perspective [7728-59]

S. Venugopal Rao, Univ. of Hyderabad (India)

772810 Single and dual wavelength pumped composite chalcogenide-tellurite microstructured fiber parametric amplifier [7728-60]

C. Chaudhari, T. Suzuki, Y. Ohishi, Toyota Technological Institute (Japan)

$77281 \mathrm{P} \quad$ Supercontinuum emission from tightly focused femtosecond pulses in air: beyond intensity clamping [7728-61]

P. Prem Kiran, S. Bagchi, Tata Institute of Fundamental Research (India) and Univ. of Hyderabad (India); S. Rama Krishnan, Sri Sathya Sai Univ. (India); C. L. Arnold, Lab. d'Optique Appliquee, CNRS, Ecole Polytechnique (France); G. Ravindra Kumar, Tata Institute of Fundamental Research (India); A. Couairon, Ctr. de Physique Theorique, CNRS, Ecole Polytechnique (France)

$77281 \mathrm{~T} \quad$ Soliton mediated quantization transmission in shallow Bragg-gratings [7728-65]

F. Eilenberger, The Univ. of Sydney (Australia) and Friedrich Schiller Univ., Jena (Germany);

C. M. de Sterke, B. J. Eggleton, The Univ. of Sydney (Australia)

$77281 \mathrm{~V} \quad$ Ultrafast Bessel beams for high aspect ratio taper free micromachining of glass [7728-67] M. K. Bhuyan, F. Courvoisier, P.-A. Lacourt, M. Jacquot, L. Furfaro, FEMTO-ST Institute, CNRS, Univ. de Franche-Comté (France); M. J. Withford, Macquarie Univ. (Australia); J. M. Dudley, FEMTO-ST Institute, CNRS, Univ. de Franche-Comté (France)

7728 1W Generation of ultrafast Bessel micro-beams and applications to laser surface nanoprocessing [7728-68]

F. Courvoisier, M. Jacquot, P.-A. Lacourt, M. Bhuyan, L. Furfaro, R. Ferrière, J. M. Dudley, FEMTO-ST Institute, CNRS, Univ. de Franche-Comté (France)

$77281 \mathrm{X} \quad$ Reconciling expressions for terahertz generation by bulk optical rectification [7728-69] C. S. Bleasdale, R. A. Lewis, Univ. of Wollongong (Australia)

7728 1Y Terahertz time-domain spectroscopy of nematic liquid crystals [7728-70] E. M. Pogson, R. A. Lewis, Univ. of Wollongong (Australia); M. Koeberle, R. Jacoby, Technische Univ. Darmstadt (Germany)

772812 Measurement of inverse Faraday effect in NiO using ultrashort laser pulses [7728-71]

K. Kuroda, T. Satoh, S.-J. Cho, R. lida, T. Shimura, The Univ. of Tokyo (Japan)

Author Index 
Downloaded From: https://www.spiedigitallibrary.org/conference-proceedings-of-spie on 26 Apr 2023

Terms of Use: https://www.spiedigitallibrary.org/terms-of-use 


\title{
Conference Committee
}

\author{
Symposium Chairs \\ Francis Berghmans, Vrije Universiteit Brussel (Belgium) \\ Ronan Burgess, European Commission (Belgium) \\ Jürgen Popp, Institut für Photonische Technologien e.V. (Germany) \\ Peter Hartmann, SCHOTT AG (Germany) \\ Hugo Thienpont, Vrije Universiteit Brussel (Belgium)
}

Conference Chairs

Benjamin J. Eggleton, The University of Sydney (Australia)

Alexander Luis Gaeta, Cornell University (United States)

Neil G. R. Broderick, University of Southampton (United Kingdom)

\section{Program Committee}

Arnaud Couairon, École Polytechnique (France)

Richard M. De La Rue, University of Glasgow (United Kingdom)

Christophe Dorrer, University of Rocheser and Laser Energetics, Inc. (United States)

John M. Dudley, Université de Franche-Comté (France)

Majid Ebrahim-Zadeh, ICFO - Instituto de Ciencias Fotónicas (Spain)

John D. Harvey, The University of Auckland (New Zealand)

Yuri S. Kivshar, The Australian National University (Australia)

Thomas F. Krauss, University of St. Andrews (United Kingdom)

Colin J. McKinstrie, Alcatel-Lucent (United States)

Leif K. Oxenløwe, Technical University of Denmark (Denmark)

David J. Richardson, University of Southampton (United Kingdom)

John E. Sipe, University of Toronto (Canada)

Session Chairs

1 Past and Future of Nonlinear Optics

Neil G. R. Broderick, University of Southampton (United Kingdom)

2 Semiconductor Based Nonlinear

Richard M. De La Rue, University of Glasgow (United Kingdom)

3 Photonic Crystal Fibres

Neil G. Broderick, University of Southampton (United Kingdom)

$4 \quad$ Slow Light Effects

John M. Dudley, Université de Franche-Comté (France) 
$5 \quad$ New Frontiers in Nonlinear Optics

Stéphane Coen, The University of Auckland (New Zealand)

$6 \quad$ Nonlinear Optics for Telecommunications

Benjamin J. Eggleton, The University of Sydney (Australia)

7 Pulse Generation and Manipulation

Neil G. R. Broderick, University of Southampton (United Kingdom)

8 Supercontinuum Generation

Neil G. R. Broderick, University of Southampton (United Kingdom)

9 Nonlinear Photonic Crystals

Daniele Faccio, Università degli Studi dell'Insubria (Italy)

10 Novel Nonlinear Effects

Richard M. De La Rue, University of Glasgow (United Kingdom) 